\title{
Interação reflexiva como paradigma transversal para a criatividade, educação musical e musicoterapia*
}

\author{
Reflective Interaction as a paradigm intersecting creativity, \\ musical education and music therapy
}

Anna Rita Addessi** annarita.addessi@unibo.it

Luisa Bonfiglioli*** luisa.bonfiglioli@unibo.it

Tradução:

Rosane Cardoso de Araújo**** rosanecardoso@ufpr.br*

\footnotetext{
* Texto traduzido do livro "Musicalità e pratiche inclusive - II mediatore musica fra educazione e benessere", organizado por Elena Malaguti, 2017. Autorizado pela Editora Erickson.

** Professora Associada em Musicologia. Professora de metodologia de educação musical e de educação sonora na Escola de Psicologia e Ciências da EducaçãodaUniversidadedeBolonha(Itália). Doutoraem Musicologia. Realizoupós-doutorado em Psicologia da Música pela Universidadede Bolonha.

*** Doutora em Psicologia Geral e Clínica, musicoterapeuta e professora tutora na Escola de Psicologia e Ciências da Educação da Universidade de Bolonha (Itália).

**** Professora Associada da Universidade Federal do Paraná (UFPR). Docente nos cursos de graduação e pós-graduação em música. Bolsista de produtividade do CNPq e coordenadora do Programa de Pós-Graduação em Música da UFPR. Doutora em Música pela Universidade do Rio Grande do Sul. Realizou pós-doutorado pela Universidade de Bolonha (Itália).
} 


\section{Resumo}

O escopo deste estudo é apresentar o paradigma da "interação reflexiva" criado no contexto dos estudos sobre a interação homem-máquina e utilizado no âmbito do Projeto MIROR- Musical Interaction Relying On Reflection. Através deste projeto, foi construída uma plataforma tecnológica musical para crianças, incluindo atividades de improvisação, criação e movimento corporal, aplicando o paradigma de interação reflexiva no campo da criatividade musical e no campo da criatividade motora infantil. $O$ foco deste texto é destacar o paradigma de interação reflexiva na interseção entre educação musical e musicoterapia, como um quadro teórico efetivo para fortalecer traços comuns a ambas as áreas, tanto do ponto de vista da aplicação teórica quanto da experiência prática.

Palavras-chave: : Interação reflexiva; Projeto MIROR; Musicoterapia; Educação Musical; Crianças.

\section{Abstract}

The scope of this study is to present the "reflexive interaction" paradigm, created in the context of studies about human and machine interaction, and used within the scope of the MIROR Project - Musical Interaction Relying On Reflection. In this project, a musical technology program was created for children, which included improvising and creative body movement activities, , applying the reflexive interaction paradigm in the field of musical creativity and in the field of children's motor creativity. The focus of this text is to highlight the reflexive interaction paradigm at the intersection between music education and music therapy as an effective theoretical framework to strengthen traits common to both areas, both from the point of view of theoretical application and practical experience.

Keywords: Reflexive Interaction; MIROR Project; Music Therapy; Music Education; Children. 


\section{Introdução}

Neste ensaio será introduzido o paradigma da "interação reflexiva", um novo paradigma científico nascido no contexto dos estudos de interação homem-máquina, desenvolvido e implementado por meio de novas tecnologias, utilizadas para instigar a criatividade infantil, musical e motora, no âmbito do Projeto MIROR ${ }^{1}$ - Musical Interaction Relying On Reflexion (Interação Musical com base na Reflexão - ver Addessi et al. (2013). No ensaio será destacado e discutido o potencial educativo e musicoterápico deste paradigma, tanto do ponto de vista teórico quanto do ponto de vista empírico e experimental. Vamos apresentar uma introdução sobre os Sistemas Musicais Interativos-Reflexivos (SMIR), os fundamentos teóricos do paradigma da interação reflexiva, seus valores pedagógicos e seu potencial musicoterápico. A apresentação teórica será ilustrada por resultados experimentais obtidos ao longo de dez anos de pesquisa que permitiram, como produto final, a implementação da Plataforma MIROR, um sistema para desenvolver criatividade musical e motora das crianças (ADDESSI, 2013). O objetivo deste ensaio é destacar o paradigma de interação reflexiva na intersecção entre a educação musical e a musicoterapia, como um marco teórico eficaz para fortalecer traços comuns às duas áreas, tanto do ponto de vista da aplicação teórica quanto da experiência prática.

\section{Sistemas musicais interativos-reflexivos}

O estudo do paradigma da interação reflexiva iniciou há alguns anos. Pachet (2003) elaborou o Continuator, um sistema que produzia respostas em tempo real imitando o estilo de um músico quando este tocava um teclado - um tipo de alter ego com o qual o músico interagia em um dueto musical envolvente. O pressuposto que orientou o design do Continuator foi permitir que o usuário (músicos adultos) manipulasse uma cópia virtual de si mesmo durante a realização de improvisações musicais. Na Fig. 1 é possível ver um exemplo deste sistema: a entrada produzida ao tocar um teclado conectado ao MIROR-Impro (uma versão melhorada/expandida do Continuator, implementada no projeto MIROR Europeu - Fig. 1a) segue de uma resposta do sistema (Fig. 1b) que repete a entrada musical feita pelo usuário com variações rítmico-melódicas. Essa resposta é percebida pelo usuário como uma espécie de "espelho sonoro" de si mesmo.

$1 \quad$ MIROR (Musical Interaction Relying On Reflexion), projeto cofinanciado pela Comunidade Europeia no tema TIC (Tecnologias de Informação e Comunicação) do 7. Programa de Pesquisa e Desenvolvimento da União Europeia (Commission `s Seventh Framework Programme) (7PM / 2007-2013). Para mais informações, visite o site oficial do projeto: <www.miroproject.eu>. 

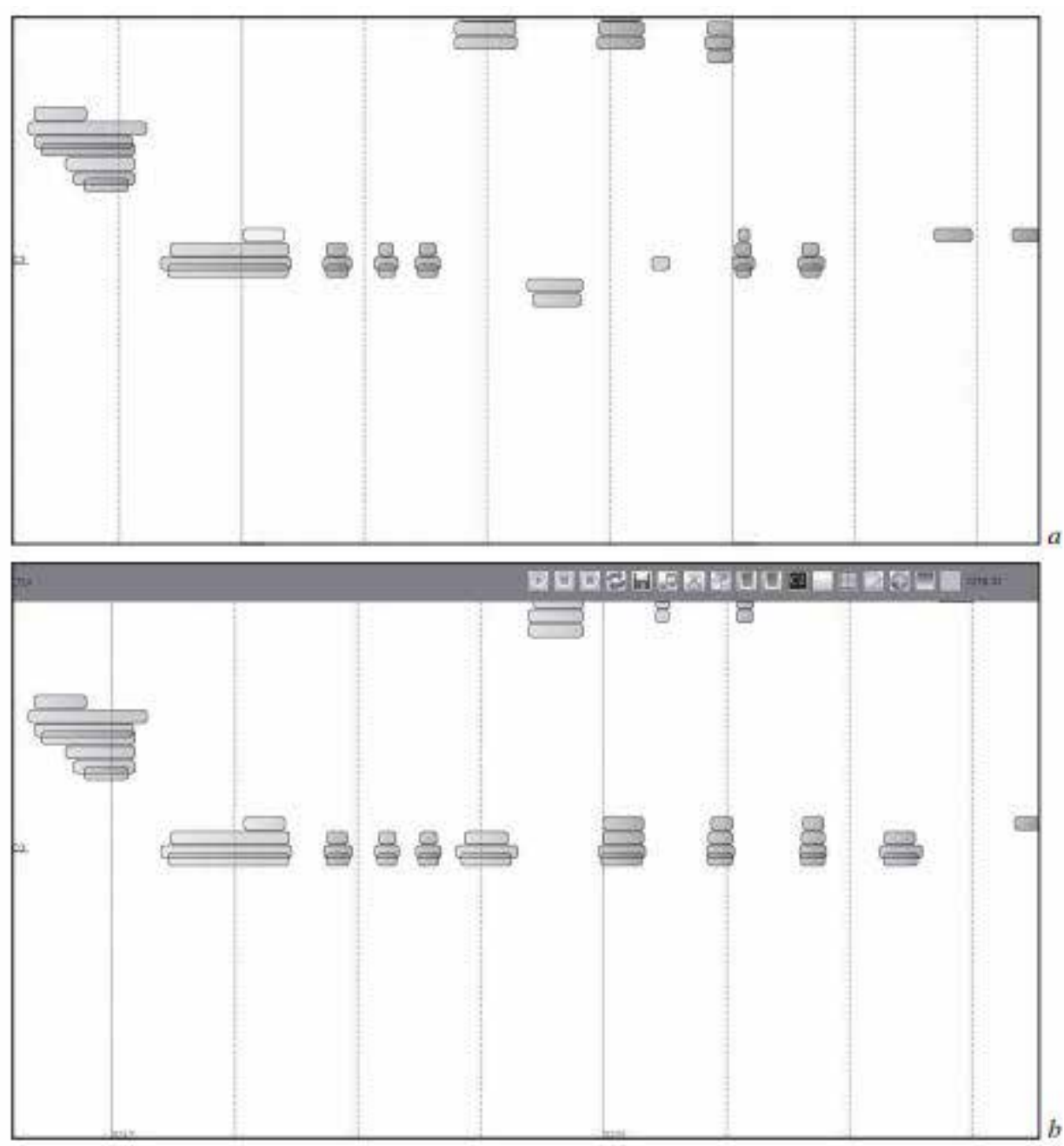

Fig. 1 - Representação gráfica de um excerto tocado por uma criança de 4 anos (1a), seguido da resposta gerada pelo MIROR-Impro (1b). A duração dos sons é representada horizontalmente; a altura dos sons está na direção vertical. É possivel observar que a resposta do sistema é uma repetição com variações rítmico-melódicas do excerto executado pela criança.

Pachet (2006, p. 360) definiu SMIR como uma "classe de sistemas interativos na qual os usuários podem interagir com cópias virtuais de si mesmos, ou, pelo menos, com agentes que têm uma capacidade mimética, que possam se envolver de maneira orgânica", e identificou uma série de recursos que caracteriza esses sistemas. Esses recursos podem ser listados da seguinte maneira:

i) Similaridade ou efeito de espelho, ou seja, os SMIRs "produzem sons como os que o usuário é [...] capaz de produzir (Id);

ii) Agnosticismo, "nenhuma informação musical pré-programada é dada ao sistema" (Id);

iii) Maior complexidade, "o aprendizado complexo garante que [o sistema] está evoluindo continuamente [...], é uma maneira de fornecer ao sistema sensibilidade orgânica, típica dos sistemas naturais abertos" (Ibidem, p. 361);

iv) Finalmente, um último recurso refere-se ao modo básico de reprodução de SMIRs, que consiste em um modo particular de alternâncias de turno entre o usuário e o sistema, regido por três princípios fundamentais: 1. A realização de uma análise 
automática, feita pelo sistema, ao final da frase interpretada pelo usuário; 2. A geração de uma frase elaborada pelo sistema cuja duração é igual à duração da última frase interpretada pelo usuário; 3. A prioridade de execução dada ao usuário, o que significa que, se o usuário começar a tocar enquanto o sistema ainda estiver sendo reproduzido, o sistema para e retoma ao passo 1 acima (PACHET, 2004).

\section{A interação da criança em um ambiente reflexivo}

O primeiro protótipo do SMIR, o Continuator, foi criado para músicos adultos. As experiências e os estudos exploratórios que realizamos com o Continuator e com as crianças imediatamente mostraram o potencial desses sistemas reflexivos para o desenvolvimento de experiências musicais criativas (ADDESSI; PACHET, 2005, 2006). A partir desses estudos, tomou forma o projeto europeu MIROR - Musical Interaction Relying On Reflexion, com o qual estamos aplicando o paradigma da interação reflexiva no campo da criatividade musical e, pela primeira vez, no campo da criatividade motora infantil (ADDESSI, 2013). Em experimentos realizados com SMIRs e crianças, foi possível observar que elas interagiam com o sistema, exploravam o teclado (acoplado ao sistema), inventavam sons e frases musicais, criavam formas originais de produzir som, ouviam atentamente suas produções e as respostas geradas pelo sistema, compartilhavam regras de alternância de turnos, inventavam novas regras junto ao sistema e interagiam com outros colegas improvisadores, com riqueza de expressão e participação.

Apesar da aparente simplicidade do mecanismo, de fato, os SMIRs, por seu comportamento especulativo, parecem gerar reações muito complexas, em que as crianças desenvolvem uma série de representações internas por conta própria e, por outro lado, pode-se observar que o sistema pode ajudar a criança a construir um "self musical". 0 que imediatamente chamou nossa atenção nos primeiros estudos com as crianças e o Continuator é que o comportamento interativo desses sistemas é muito semelhante ao observado entre os seres humanos, graças à sua capacidade de se comportar de forma "reflexiva", como vamos ilustrar detalhes nos próximos parágrafos.

Para entender melhor como a interação reflexiva funciona com um SMIR, observamos e descrevemos uma breve sessão de uma menina de oito anos de idade tocando num teclado conectado ao MIROR-Impro. Esta sessão foi registrada no âmbito do Projeto MIROR, durante a implementação do Protocolo Experimental no 2 (ADDESSI; ANELLI; BENGHI; FRIBERG, 2017), na cidade de Bolonha, em 2013:

Descrição da interação observada: a criança toca duas notas consecutivas, dó2 e lá2, para e aguarda a resposta do sistema. O sistema responde repetindo as mesmas notas. A menina então toca uma única nota, sol2, e o sistema responde tocando uma nota e na sequência apresenta uma variação: o dó3, introduzindo assim um registro mais agudo. A criança, seguindo a variação introduzida pelo sistema, move-se para o registro mais agudo e, por sua vez, desempenha uma variação do padrão inicial: re2-lá2-mi2-dó3, introduzindo também um padrão rítmico particular. Este evento "reflexivo" marca o início de um diálogo baseado na repetição e na variação: o padrão rítmico-melódico quanto às alturas e va- 
riações rítmicas será repetido e alterado, seja pelo sistema quanto pela menina durante a alternância dos turnos, para adquirir a forma de uma criação musical completa. Em certo ponto do diálogo, a criança começa a acompanhar a resposta do sistema com movimentos de braços sincronizados com o padrão rítmico-melódico, resultando em uma espécie de composição musical-motora.

Neste exemplo, é possível observar os elementos fundamentais da interação reflexiva com um sistema interativo-reflexivo:

1. A interação é baseada na alternância de turnos;

2. A resposta do sistema tem uma duração de tempo equivalente à última frase desempenhada pela criança, dando, assim, origem a um tempo de resposta regular;

3. A atenção da criança concentra-se mais na interação musical com o sistema quando o sistema imita a sentença desempenhada pela criança;

4. O diálogo que emerge entre a menina e o sistema MIROR-Impro não é predeterminado pela máquina e não é apenas realizado pela criança, mas é construído pela criança juntamente com o sistema;

5. A corregulação é baseada em um mecanismo de repetição e variação contínua entre as frases musicais tocadas pela criança e pelo sistema;

6. Os dois parceiros podem se imitar mutuamente;

7. A criança reconhece a resposta do sistema como uma imitação de sua produção musical.

Essas observações nos mostram como o sistema reflexivo pode imitar e variar as frases musicais propostas pela menina e como esse comportamento despertou na criança surpresa, curiosidade e interesse. Tais reações a encorajam a passar da execução casual de duas notas para uma elaborada sequência de padrões melódicos rítmicos e frases musicais, em um diálogo musical com o sistema, que é também interpretado pelo movimento do corpo, especialmente dos braços.

\section{Uma abordagem sistemático-musicológica do paradigma da interação reflexiva}

Com esse tipo de observações empíricas, várias teorias foram consideradas para explicar o comportamento humano observado durante a interação com os sistemas reflexivos, permitindo formular, assim, um quadro teórico sobre a interação reflexiva, com implicações psicopedagógicas (ADDESSI, 2014). Do ponto de vista sistemático-musicológico (ver BARONI, 1985; LEMAN, 2008), podemos argumentar que os temas do "espelho" e do "espelho sonoro" caracterizaram a cultura ocidental há muito tempo. As referências vão desde o mito de Eco e Narciso (Ovídio, 43 a.C.-18, Metamorphoseon libri XV) aos efeitos de eco da música renascentista e barroca. Na Teoria dos Afetos (Vincenzo Galilei, Dialogo della musica antica et della moderna, Firenze, 1581) e na Affektenlehre - Doutrina dos afetos (Athanasius Kircher, Musurgia Universalis, Roma, 1650), é possível encontrar outra maneira de pensar e de reconhecer o poder do "reflexivo" da música, isto é, de representar com sons os afetos humanos e suscitar, de forma empática, tais emoções no ouvinte. 
A principal característica da interação reflexiva é o mecanismo de repetição e variação. Na interação reflexiva, algo é repetido e variado durante a interação, através de um contínuo processo de imitação e variação. Esta característica também é a base de algumas correntes recentes de análise musical da natureza semiótica (por exemplo, RUWET, 1966) e de teorias sobre a percepção de semelhanças em ouvir música (por exemplo, DELIĖGE, 2001; TOIVIAINEN, 2007). Estudos recentes em psicologia e neurociência sugerem que este mecanismo desempenhe um papel importante no desenvolvimento da musicalidade durante a infância e que seja um dos fundamentos ontológicos da musicalidade humana (ver CROSS, 2008; DISSANAYAKE, 2000; IMBERTY, 2005; MALLOCH; TREVARTHEN, 2009a; MITHEN, 2005; STERN, 2004). Os processos de imitação, reconhecimento da imitação, autoimitação, repetição e variação, de fato, são desenvolvidos nos primeiros meses de vida e estruturam o Eu da criança e sua interação com o ambiente circundante (ver GRATIER e APTER-DANON, 2008; NADEL; BUTTERWORTH, 1999; PAPOUSĚK, 2007). Em nosso estudo anterior, desenvolvido em um contexto naturalista sobre a interação vocal entre crianças de oito meses de idade e o pai ou a mãe, por exemplo, pudemos observar que a criança é vocalmente mais ativa quando o pai imita as vocalizações da criança apresentando variações espontâneas (ADDESSI, 2009). Anzieu (1996) define esse tipo de experiência de infância como o “Invólucro Sonoro do Ser", onde o Eu é descrito como o primeiro embrião da personalidade, percebido como unidade e individualidade e cujo desenvolvimento está associado à expressão de uma das formas mais arcaicas de repetição: o eco.

Outro aspecto que podemos derivar desta literatura é a importância da interação reflexiva como um processo dinâmico que se desenvolve ao longo do tempo, no decorrer das interações iniciais entre mãe e bebê: a experiência de repetição e variação é realizada por meio de condições afetivas e emotivas amodais que Stern (2004) definiu como "contornos afetivos" ou "afetos vitais". É interessante notar que, ao descrever a estrutura desse tipo de experiência de "eco" entre a mãe o bebê, Stern utilizou três categorias que fazem parte da própria natureza musical: forma, intensidade e ritmo, usando como metáfora o conceito de "Afinação afetiva", que é o tipo de acordo criado entre os músicos de um quarteto de cordas, por exemplo, quando tocam juntos.

Malloch e Trevarthen (2009a), a partir da observação de ritmos e imitações nas interações mãe-filho, finalmente teorizaram o conceito de "pulso" e a natureza musical da comunicação humana, chamando-a de "musicalidade comunicativa". O mesmo fenômeno de corregulação observado na interação entre crianças e SMIRs pode ser analisado de acordo com as dinâmicas inter-relacionais descritas por Fogel (2000) na comunicação humana, bem como com base em estudos recentes de comunicação musical, como foi observado, por exemplo, entre músicos durante uma improvisação de jazz (ver SAWYER, 1999; GRATIER, 2008).

No quadro teórico-sistemático descrito acima, portanto, o paradigma de interação reflexiva, que afirmamos surgir também no campo da interação humano-máquina, se amplia, estendendo-se ao longo da história ocidental e estética musical ocidental e nos comportamentos humanos, observáveis desde o nascimento até alcançar a 
esfera ontológica da comunicação humana.

Nas últimas décadas, o mecanismo de repetição e variação encontrou declinações interessantes também à luz de estudos recentes no campo da neurociência. Zatorre (2012), por exemplo, destacou alguns mecanismos neurais e cognitivos que permitem transformar e manipular representações mentais musicais preexistentes. Margulis (2014) traçou uma visão interessante e abrangente da importância da repetição na música, tanto do ponto de vista linguístico quanto do ponto de vista cognitivo-neurobiológico. A capacidade de replicar o comportamento de outros pode encontrar seus fundamentos neurobiológicos no mecanismo do sistema neurônio espelho, uma rede de neurônios que se tornam ativos durante a execução e/ou observação de ações (RIZZOLATTI; FADIGA; FOGASSI; GALLESE, 2002). Esses pesquisadores levantam a hipótese de que existe um mecanismo evolutivo, chamado de ressonância, através do qual as descrições visuais dos comportamentos motores são diretamente correspondentes aos movimentos do observador que observa os mesmos comportamentos. No campo da cognição musical incorporada, Leman (2007, p. 91) ressalta que "há evidências [...] de que os neurônios espelhos são amodais no sentido de que podem codificar o espelhamento de múltiplos canais sensoriais". Portanto, uma interação reflexiva através do canal auditivo, como ocorre durante a interação com MIROR-Impro, estimularia um mecanismo de ressonância na criança em áreas motoras do cérebro. Este campo de estudo e sua aplicação nas ciências da educação ainda devem ser mais explorados.

\section{Os conceitos pedagógicos}

A hipótese básica do projeto MIROR é que a interação reflexiva estimula a criatividade e a expressividade musical na criança, favorecendo e apoiando os processos de aprendizagem da música. Graças à sua capacidade de reproduzir "música humana" e de evoluir de forma orgânica com o usuário, os SMIRs traduzem conceitos de desenvolvimento, criatividade e conceitos de aprendizagem em design tecnológico. Em geral, é possível dizer que os SMIRs estão numa perspectiva socioconstrutivista de aprendizagem. No entanto, um dos resultados da pesquisa realizada no âmbito do projeto MIROR é que o paradigma de interação reflexiva não pode ser descrito de forma abrangente por teorias existentes. Este paradigma é, de fato, uma nova perspectiva pedagógica no campo da interação criança-computador, e os SMIRs representam uma aplicação original no campo da tecnologia de aprendizagem.

A eficácia pedagógica do conceito de interação reflexiva tem sido demonstrada por meio de estudos realizados inicialmente com crianças e o Continuator (ADDESSI; PACHET, 2005, 2006; FERRARI; ADDESSI, 2014) e, em seguida, no projeto MIROR, com os três aplicativos implementados: o MIROR-Impro (PACHET; ROY; BARBIERI, 2011), dedicado à improvisação; o MIROR-Compo, dedicado à composição musical, e o MIROR Body-Gestor, (VOLPE et Al, 2012), dedicado à criatividade motora e musical (ver ADDESSI; FERRARI; CARUGATI, 2015; ADDESSI; MAFFIOLI; ANELLI, 2015; ADDESSI; ANELLI; BENGHI; FRIBERG, 2015; ALEXAKIS; KHATCHATOUROV; TRIANTAFYLLAKI; ANAGNOSTOPOULOU, 2013; ANAGNOSTOPOULOU; ALEXAKIS; TRIANTAFYLLAKI, 2012; CARDO- 
SO DE ARAUJO; ADDESSI, 2014; FERRARI; ADDESSI, 2013; ROWE; TRIANTAFYLLAKI; ANAGNOSTOPOULOU, 2014; TRIANTAFYLLAKI; ANAGNOSTOPOULOU; ALEXAKIS, 2012; WALLERSTEDT; LAGERLOF, 2011). Estes estudos também relatam uma série de detalhes críticos a partir dos quaisl foi possível elaborar listas de requisitos para melhorar essas tecnologias em suas capacidades de ensino (para uma visão geral sobre os resultados, ver Projeto MIROR - Relatório Final, 2013²). Na conclusão do projeto, os resultados de estudos experimentais foram traduzidos para uma série de práticas educativas e criativas, realizáveis com a Plataforma MIROR, destinadas a crianças e adultos e que são aplicáveis em contextos formais e informais de aprendizagem musical e motora (ADDESSI, 2015).

Foi possível observar que a interação reflexiva com esses sistemas estimula o experiências profundas de diálogo e comunicação nas crianças, que são a base de suas experiências criativas e expressivas. Os SMIRs permitem que a criança dê voz e forma musical às suas emoções e à sua imaginação, atendendo à necessidade de comunicação e socialização, permitindo a construção conjunta de diálogos sonoros e criações motoras. Em nossa opinião, o elemento expressivo na interação reflexiva da criança com SMIRs é prioritário sobre a aprendizagem. Precisamente por esta razão, acreditamos que esses sistemas possam ser um instrumento eficaz para a aprendizagem musical e desenvolvimento motor, porque, de acordo com Baroni (1997, p. 141), acreditamos na "necessidade absoluta de enfatizar o momento de expressão sobre a aprendizagem: e isso não só porque a construção de objetos expressivos pode ser considerada como o principal fim, mas também porque é a única motivação válida e persuasiva das atividades de aprendizagem".

Na prática, os SMIRs recordam o conceito vygotskiniano de "Zona de Desenvolvimento de Proximal" (VYGOTSKY, 1962), estabelecendo uma interação de aprendizagem entre as crianças e o próprio sistema. Esta característica permite que as crianças desenvolvam autorregulação e comportamentos de autoiniciativa, ou seja, uma abordagem centrada na própria criança. No entanto, tais sistemas não se comportam como adultos que, no conceito vygotskiano, desempenham o papel de parceiro mais competente e, portanto, de tutor. No caso dos SMIRs, eles não possuem maior competência musical do que as crianças, pelo contrário, aprendem com as próprias crianças durante o curso da interação. A interação entre a criança e um sistema musical interativo-reflexivo é mais semelhante, portanto, ao modelo de interação entre pares.

O quadro pedagógico do SMIR caracteriza-se, portanto, pelos seguintes elementos constituintes:

1. Prioridade dada à criança e aprendizagem centrada no aluno: através do processo de corregulação, na interação reflexiva, o foco principal na interação não é o produto musical final, mas a pessoa envolvida na interação;

2. Adaptabilidade: o sistema é constantemente e organicamente adaptado ao estilo musical e motor de cada criança;

3. O "método de ensino" do SMIR é baseado na alternância de turnos, na regularidade de turnos, em estratégias de reflexão, modeling e scaffolding (BRUNER, 1983; 
VYGOTSKY, 1962), sobre motivação intrínseca, jogo colaborativo e atenção conjunta;

4. Aprendizagem "reflexiva": diferentemente do que acontece na aprendizagem por imitação, durante uma interação reflexiva, o mecanismo de aprendizagem é desencadeado pela experiência de "ser imitado";

5. Ausência de objetivos predefinidos: os SMIRs não estão programados com objetivos fixos, como é o caso de muitos softwares de treinamento auditivo (ear-training) para o reconhecimento de acordes etc. Com o SMIR, os produtos musicais são o resultado da interação em si e devem ser construídos conjuntamente pela criança em interação com o sistema;

6. Propriedade da transparência e reflexão (FOLKESTAD; HARGREAVES; LINDSTRÖM, 1998): os SMIRs possuem as propriedades da transparência (as crianças, de fato, só interagem com SMIR predominantemente tocando ou através do movimento) e a reflexão no sentido de que o próprio sistema ajuda o usuário a entender as regras de interação;

7. Fator de distância (BERTOLINI; DALLARI, 2003): observamos que as crianças são capazes de interromper a interação quando querem, preservando, assim, o fator "distância" entre o eles e o sistema, um fator de extrema importância, seja do ponto de vista estético quanto do ponto de vista pedagógico;

8. $A$ atratividade: SMIRs evitam a monotonia da mera repetição, com a introdução de variações contínuas e "erros", atuando como "máquinas imperfeitas";

9. Jogo colaborativo: o duplo papel de um SMIR, como parceiro virtual e tutor, parece aumentar a criatividade musical das crianças, tanto na exploração como na improvisação e na socialização com pares;

10. Improvisação Musical: as observações revelaram vários modelos de diálogo musical e improvisações rítmico-melódicas, experiências compartilhadas de sincronização no mesmo pulso, criação de padrões rítmico-melódicos e frases musicais baseadas em imitação, repetição, alternância e contraste;

11. Identidade e estilo musical: a resposta especular do sistema suporta e fortalece o desenvolvimento da identidade pessoal e do estilo musical de cada criança, permitindo que cada criança se expresse e desenvolva uma linguagem musical original e individual, de acordo com seu estilo comunicativo e expressivo. Nas experiências músico-motoras, estão envolvidas também a identidade e o estilo gestual (de movimento) de cada criança;

12. Audição reflexiva: um aspecto pedagógico que é particularmente interessante na interação reflexiva é a qualidade de permitir observar a escuta da criança sobre sua própria produção, mediada pelo elemento interativo que incentiva as crianças a comparar suas peças com a resposta (e nova proposta de sistema), para identificar repetições e diferenças. Este aspecto parece ser particularmente importante, pois, como salientado por muitos autores (ver, entre outros, DELALANDE 1993; TAFURI 1995; MCPHERSON, 2005), ouvir atentamente as próprias produções musicais é um dos principais objetivos da educação musical.

Finalmente, a aplicação do terceiro protótipo, o MIROR-Body Gesture, explora e amplifica uma série de elementos de análise de gestos, elaborados pelo teórico da dan- 
ça Rudolf Laban (1980). As teorias e ferramentas desenvolvidas por Laban têm sido amplamente utilizadas na educação para o desenvolvimento motor e da dança, também em relação à educação musical (ver ZAGATTI, 2004). Com o projeto MIROR pretendemos usar esse quadro teórico e seus efeitos de aplicação a partir do contexto da dança educativa para desenvolver habilidades musicais e motoras de crianças através de tecnologias reflexivas (ver ADDESSI; MAFFIOLI; ANELLI, 2015).

\section{Teoria da interação reflexiva e do fluxo}

Em nossos estudos sobre a interação das crianças com os SMIRs, a Teoria do Fluxo (CSIKSZENTMIHALYI, 1996) provou ser uma ferramenta teórica e experimental eficaz, pois oferece novos critérios que ajudam a analisar quantitativa e qualitativamente índices observáveis nos processos criativos e estados de bem-estar (alegria/satisfação) resultantes da interação criança-máquina. O estado de fluxo foi definido por Csikszentmihalyi como uma "experiência ótima" resultante do equilíbrio percebido entre os objetivos e as habilidades que o sujeito possui para alcançar esses objetivos. Esse "estado de bem-estar" é considerado a base dos processos criativos. O estado do fluxo é caracterizado pela presença de níveis elevados de uma série de variáveis, que são: atenção concentrada, feedback claro e imediato, objetivos claros, prazer, controle de situação, nenhuma preocupação com falhas, desaparecimento de autoconsciência, modificação na percepção do tempo. De acordo com a teoria de Csikszentmihalyi, além do estado de fluxo, outros estados emocionais podem ser observados, dependendo da intensidade e presença diferentes de cada uma das variáveis: aborrecimento (quando os objetivos são menores do que as habilidades possuídas), ansiedade (quando os objetivos são superiores às habilidades possuídas), excitação, controle, preocupação, relaxamento e apatia. Nossos estudos com crianças e SMIRs mostram que a interação reflexiva, com o uso de SMIRs, aumenta a experiência de fluxo e, portanto, de bem-estar e criatividade (ADDESSI; FERRARI; CARUGATI, 2015). As experiências demonstram claramente que as crianças estão envolvidas em atividades focadas, tanto quando tocam, quanto quando ouvem; elas brincam com o sistema de forma automotivada, sem a necessidade de um estímulo externo; as crianças têm o controle da situação na maior parte do tempo. Elas imediatamente entendem que podem interromper o sistema quando querem, ou inventam novas regras.

Os resultados mais óbvios do experimento (tempo prolongado de atenção, escuta autotélica, efeito "Aha!!") podem ser vinculados à motivação intrínseca que encoraja as crianças a interagirem com o sistema. As crianças não recebem metas para alcançar, exceto para tocar o tempo que quiserem: os objetivos são criados espontaneamente durante a própria interação. Por exemplo, em muitas sessões observamos que as crianças estão tentando ensinar ao sistema algumas passagens que acabaram de tocar. 


\section{Contextos terapêuticos e de reabilitação}

Estudos realizados até o momento atual mostraram que a interação reflexiva também pode ser um dispositivo versátil para aprimorar e estimular o comportamento expressivo e comunicativo em situações de deficiência e/ou situações em que é importante encorajar a inclusão. A "experiência de fluxo" gerada pela interação com aplicativos MIROR (ver ADDESSI; FERRARI; CARUGATI, 2015) promove "bem-estar" e "experiência ótima", sugerindo um potencial terapêutico e de reabilitação efetivo. A interação reflexiva estimula e ativa processos interativos que envolvem profundamente a pessoa, bem como áreas específicas da ressonância cerebral. Nesse sentido, a interação reflexiva estimulada pelos SMIRs pode ser um dispositivo importante para tratamentos clínicos e se tornar uma ferramenta útil para o terapeuta. Por exemplo, Nadel (2002) salienta que os processos de imitação e de reconhecimento da imitação, ou seja, os processos que vimos, são a base da interação reflexiva e são cruciais para a compreensão da síndrome do autismo. De acordo com Rizzolatti et al. (2002), o autismo pode ter uma base neurobiológica na falha dos neurônios espelho. Os resultados do experimento de Gergely e Watson (apud NADEL, 2002) e Nadel (2002) sobre a preferência pela perfeita contingência temporal dos sistemas pelas crianças com autismo são importantes para nosso propósito. A presença de uma contingência temporal fixa, que caracteriza a interação com SMIRs e que os caracteriza como máquinas perfeitas, juntamente com suas respostas baseadas, por sua vez, em variações "imperfeitas" sobre o ritmo, a melodia, o timbre etc., poderiam dar espaço a um dispositivo que tem como qualidade a "transicionalidade", enquanto, no diálogo entre a criança e o sistema, fica preservado, seja o caráter da máquina "perfeita" e mais previsível, preferido por crianças com autismo, quanto o caráter imperfeito de contingência, que torna esses sistemas mais "humanos" e próximos aos comportamentos sociais dos indivíduos.

O uso terapêutico da interação reflexiva com SMIRs ainda é inexplorado e novas investigações estão em andamento no âmbito do projeto MIROR, com pessoas com autismo, síndrome de Down e contextos relacionados à música na escola (ANAGNOSTOPOULOU et al., 2012; LEONI, 2011; BONFIGLIOLI, 2015; BONFIGLIOLI \& ADDESSI, no prelo a).

Em geral, pode-se afirmar que a interação reflexiva, com as qualidades que a caracterizam (Fig. 2), em particular os mecanismos de espelhamento, repetição e variação, corregulação, temporização regular, turnos alternados etc., podem representar, com e sem tecnologias, um paradigma transversal para a criatividade, educação, aprendizagem e para os contextos de inclusão - áreas complementares na experiência humana, embora com diferentes objetivos - graças ao fato de captar os mecanismos subjacentes da natureza da identidade humana, que encontramos na esfera da arteterapia e da musicoterapia. 

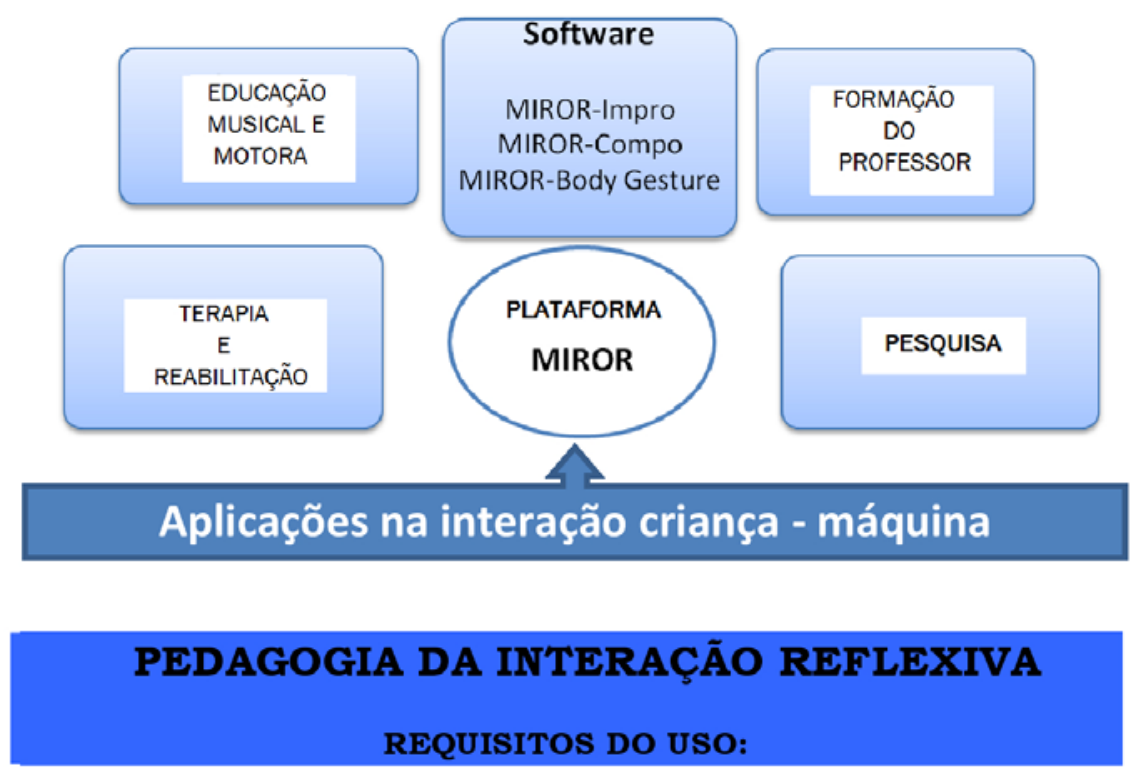

\begin{tabular}{|c|c|c|}
\hline $\begin{array}{c}\text { Modos de interação } \\
\text { Espelhamento } \\
\text { Alternância de turnos } \\
\text { Tempo regular dos } \\
\text { turnos } \\
\text { Contingência Temporal } \\
\text { Role-taking } \\
\text { Corregulação }\end{array}$ & $\begin{array}{c}\text { Experiência do } \\
\text { ouvinte } \\
\text { Interagir com cópia } \\
\text { virtual de si mesmo } \\
\text { Imitação e auto- } \\
\text { imitação } \\
\text { Life cycle } \\
\text { Fluxo } \\
\text { Invenção de regras } \\
\text { Atenção compartilhada } \\
\text { Pensar nos sons } \\
\text { Atividade autorregulada } \\
\text { Motivação intrinseca }\end{array}$ & $\begin{array}{c}\text { Conceitos Pedagógicos } \\
\text { Aprendizagem centrada na } \\
\text { criança } \\
\text { Reespelhamento } \\
\text { Scaffolding-Modeling } \\
\text { Adaptativo } \\
\text { Sem objetivos fixos } \\
\text { Trasparência-Reflexão } \\
\text { Fator de distância } \\
\text { Improvisação } \\
\text { Escuta reflexiva } \\
\text { Prática colaborativa } \\
\text { Criatividade }\end{array}$ \\
\hline
\end{tabular}

Fig. 2 - Resumo gráfico da estrutura teórica do paradigma de interação reflexiva (Fonte: ADDESSI, 2015)

\section{A música como mediadora na musicoterapia e educação musical: quais ele- mentos de continuidade são possíveis?}

Como diz Bruscia (1998), o propósito geral da musicoterapia diz respeito à promoção da saúde do indivíduo. Este propósito é articulado de acordo com as necessidades específicas do paciente, ou grupo de pacientes, para quem a intervenção é projetada. É verdade que o conceito de saúde deve ser considerado como uma construção que, como afirmou a abordagem biopsicossocial (ENGEL, 1977), inclui fatores de diferentes naturezas e não se destina unicamente a um ponto de vista orgânico, como postula a abordagem biomédica. Esta consideração é ainda mais válida para situações em que a musicoterapia é muitas vezes necessária: trata-se, em muitos casos, de pacientes com deficiências muito importantes e incapacitantes que comprometem de maneira bastante significativa, seja o funcionamento da pessoa em vários níveis (afetivo, cognitivo, motor etc.), seja a possibilidade de satisfazer a integração social. Deve-se enfatizar que a música, como mediadora que facilita o desenvolvimento do relacionamento paciente-musicologista, pode ser usada em diferentes contextos. 
Observa-se, porém, que o termo "contexto" pode ser considerado pelo menos em relação a quatro critérios: 1) os níveis de intervenção (preventiva, reabilitadora, terapêutica); 2) os âmbitos nos quais a musicoterapia está inserida (educacional, hospitalar-pediátrico, neuropsiquiatria infantil etc.); 3) a centralidade de utilização do canal sonoro-musical (música em terapia e música como terapia, de acordo com a distinção proposta por Bruscia [1998]); 4) as áreas (cognitiva, sensorial, afetivo-relacional) sobre as quais a intervenção se concentra principalmente. Consequentemente, não é possível chegar a uma definição única da musicoterapia, pois, nos contextos italiano e internacional, a disciplina é caracterizada por uma diferenciação considerável, tanto do ponto de vista teórico como do ponto de vista operacional. Essa variabilidade certamente não é um limite, mas, sim, um recurso precioso que atende à necessidade de diferentes técnicas e métodos fornecidos pelas diferentes diretrizes teóricas, dependendo das necessidades clínicas particulares que cada paciente/cliente precisa.

Uma definição recente da Federação Mundial de Musicoterapia (World Federation of Music Therapy - WFMT, 2011) ilustra muito bem a complexidade da disciplina acima descrita, tanto em termos de múltiplas áreas de intervenção quanto em termos de articulação de objetivos. A WFMT enfatiza a forma como a música e os seus elementos são utilizados numa perspectiva profissional para promover intervenções em diferentes âmbitos (médico, educacional e vida cotidiana) e com diferentes sujeitos (indivíduos, grupos, famílias ou comunidades). O objetivo das intervenções é melhorar sua qualidade de vida, saúde e bem-estar e considerar diferentes aspectos (físicos, sociais, comunicativos, emocionais, cognitivos e espirituais). Finalmente, a WFMT ressalta a importância de adotar padrões profissionais - para pesquisa, educação e treinamento clínico - que não podem ser considerados no sentido absoluto, mas devem ser incluídos em contextos culturais, sociais e políticos específicos.

$\mathrm{Na}$ realidade italiana, entre os contextos em que o musicoterapeuta pode trabaIhar, a escola certamente constitui um contexto em que a intervenção do profissional da musicoterapia é cada vez mais exigida. Professores e musicoterapeutas estão envolvidos na leitura interdisciplinar de situações complexas, cada vez mais numerosas na realidade escolar italiana, daqueles alunos que não podem ser certificados com deficiência ou com déficit de atenção, mas que experimentam dificuldades de aprendizado devido a desvantagens pessoais, familiares, socioambientais e culturais. O nível de musicoterapia na área da escola é principalmente na área de prevenção (primária, secundária e terciária) e está incluído na implementação de estratégias para encorajar processos de inclusão, nos quais a música é usada como um mediador efetivo.

Em particular, a musicoterapia pode ser configurada como uma intervenção destinada a facilitar um projeto de identidade integrativa no contexto de caminhos inclusivos (BONFIGLIOLI, 2008). Essa integração pressupõe que haja, dentro do indivíduo, uma concepção entre a constituição do mundo interior e exterior que é realizada em nível espacial (que inclui a identidade do corpo e a distinção entre si e o outro), temporal (em relação à possibilidade de organizar representações estáveis ao longo do tempo) e, finalmente, social; esta última, declinação do período de integração, exige a aquisição progressiva da capacidade de articular adequadamente o processo relacional eu/outros 
e de se relacionar com o mundo exterior sem ser ameaçado em sua própria identidade (POSTACCHINI; RICCIOTTI; BORGHESI, 1998, 2014). Entende-se, portanto, que a tarefa do musicoterapeuta é fornecer, através da escuta, as condições para incentivar e promover o desenvolvimento de processos criativos e sociais (BUNT, 2012). Como observa Canevaro (2007), a análise do relacionamento instituição-instituinte (um assunto muito importante no campo disciplinar pedagógico) refere-se imediatamente ao conceito de mediador e, entre as características centrais do mediador, há a possibilidade de se considerar a pluralidade de possíveis mediadores e a consequente habilidade destes, de se conectarem (como um efeito "dominó"), resultando, assim, em uma "contenção" elástica. Nesse sentido, deve-se ressaltar que, na escola, a intervenção da musicoterapia só pode ser efetiva se inserir, como foco central, a criança ou um grupo de crianças ou de classes. A musicoterapia, necessariamente, pode ser contemplada como uma intervenção relacionada a outras intervenções (educativas e/ou reabilitativas-terapêuticas) e colocada em sinergia com elas. Considerando estas premissas, pode-se supor que a intervenção da musicoterapia visa facilitar um projeto integrador de identidade no contexto de caminhos inclusivos.

O tema da relação entre musicoterapia e educação musical é amplamente discutido em contextos culturais e profissionais europeus. Como Bunt (2003) ressalta, referindo-se, em particular, ao contexto inglês, as associações profissionais de musicoterapeutas destacam como a musicoterapia e a educação musical devem ser consideradas formas de intervenção complementares e não alternativas. Em particular, o autor sugere que a intervenção da musicoterapia no campo educacional se justifique em todos os casos em que seja necessário elaborar ações para promover a saúde, o bem-estar e o desenvolvimento social, pré-requisitos necessários para assegurar à criança um caminho adequado de aprendizagem, crescimento e desenvolvimento. Estas considerações também são válidas para o contexto específico da escola italiana. Tanto para os terapeutas musicais como para os profissionais da música, seria desejável trabalhar para um propósito comum: o uso da música para facilitar não só o aprendizado, mas também o desenvolvimento da criança na sua totalidade, de acordo com uma visão holística da sua saúde, tanto internamente como externamente no contexto escolar.

Constata-se que existem certos elementos ou temas específicos da disciplina da musicoterapia que poderiam ser um terreno comum entre os terapeutas musicais e os professores de educação musical. Entre esses tópicos é útil propor pelo menos três deles: o conceito de musicalidade e de dois temas referentes tipicamente ao campo disciplinar da psicologia, que são a comunicação não verbal e a relação entre linguagem expressiva e emoção. Em particular, deve-se considerar que, na musicalidade comunicativa ('communicative musicality'), proposta por Malloch e Trevarthen (2009b), a conotação intersubjetiva da musicalidade é enfatizada. Outro aspecto que diz respeito ao campo musicoterápico remonta à proposta feita pelos próprios autores para considerar a musicalidade como um elemento universal e inato da espécie humana que permite apreciar e produzir uma variedade infinita de formas narrativas. Os próprios autores extraem, de forma linear e consequente, a possibilidade de usar essa competência inata no campo terapêutico: "A capacidade inerente de musicalidade para transmitir e 
comunicar os elementos 'essenciais' e o significado é enraizado na musicoterapia e na terapia dança/movimento" (MALLOCH e TREVARTHEN, 2009b, p. 6). A musicoterapia e a terapia de dança/movimento podem, portanto, de acordo com os autores, ser consequência direta da conotação comunicativa e intersubjetiva da musicalidade humana.

Considerando as contribuições teóricas específicas da Infant Research (STERN, 1985; BEEBE; LACHMANN, 2003), que nos permitiram analisar em profundidade o vínculo entre aspectos não verbais da comunicação e expressão emocional na interação entre mãe-bebê, pode-se dizer que o terapeuta de música constrói a relação juntamente com o paciente através de gestos, sons, variações dinâmicas e pulsações, ritmos e tendências melódicas, a fim de facilitar a comunicação e o compartilhamento de estados emocionais profundos.

\section{Improvisação na musicoterapia de acordo com o paradigma da interação reflexiva}

Considerando-se os elementos de complexidade e heterogeneidade presentes nos modelos da musicoterapia atualmente, propostos a nível nacional e internacional ${ }^{3}$, pode-se observar que o profissional da musicoterapia, em sua prática profissional, pode se referir a uma abordagem ativa - propondo a exploração, pelo paciente/cliente, da voz e do uso de instrumentos musicais nas práticas improvisadas ou na composição de canções - ou uma abordagem receptiva - propondo ao paciente/cliente ouvir músicas mais ou menos complexas, retiradas de diferentes e variados repertórios e gêneros musicais. O que muitas vezes acontece no cenário musicoterápico é que o musicoterapeuta, dependendo das necessidades do paciente (ou grupo de pacientes), avalia a oportunidade de usar, em diferentes momentos da sessão, a improvisação e/ou a apreciação, integrando as duas abordagens (ativas e receptivas) dentro do ciclo da sessão.

Como mencionado acima, os sistemas musicais interativos-reflexivos estão na confluência entre a educação musical e a musicoterapia, e acredita-se que o paradigma da interação reflexiva possa representar um quadro teórico eficaz para fortalecer os traços, sejam teóricos, sejam práticos, comuns às duas áreas de experiência. A abordagem ativa, que envolve o uso da improvisação musical, certamente representa o contexto dentro do qual é possível analisar esta proposta mais claramente e ilustrar mais especificamente quais elementos do uso da improvisação em musicoterapia podem surgir em continuidade com o uso da improvisação, em contextos de educação musical em ambientes reflexivos. Considerando, em particular, a prática de improvisação, Bunt (1997) lembra que o termo "improvisação" deriva do latim improvisus (imprevisto) e, historicamente, no sentido musical, o termo pode ser relacionado ao típico caráter extemporâneo que a música ocidental de tradição culta manteve até o século XVIII.

No contexto da musicoterapia, os pacientes/clientes são convidados a improvisar junto com o musicoterapeuta para construir, traçar e reconhecer uma série de relações entre os elementos musicais "cocriados" durante a própria improvisação. A improvisação 
pode possuir um caráter mais ou menos direto, dependendo dos objetivos do caminho e das necessidades do paciente, mas inclui, no entanto, a criação extemporânea de formas expressivas sonoras ou de música ao tocar ou cantar (BRUSCIA, 1987). A densa rede de conexões construídas durante uma experiência de improvisação diz respeito não apenas às relações entre os sons existentes na improvisação, mas também às relações que são construídas entre os participantes da improvisação. Por conseguinte, pode-se dizer que o valor específico da improvisação musicoterápica não é o resultado da própria performance - de acordo com cânones estéticos predefinidos -, mas, sim, das conexões intermediárias e interpessoais que foram criadas durante o processo de improvisação.

No cenário musicoterapêutico, a tarefa do musicoterapeuta que improvisa junto com o paciente é, portanto, acolher e valorizar todas as contribuições musicais criadas pelo indivíduo, respondendo com autenticidade e de maneira direta. Esta maneira de trabalhar permite que o musicoterapeuta crie um espaço terapêutico seguro e concomitante (BUNT, 1997). Não se deve esquecer que graças ao fato de que, na improvisação musicoterapêutica, a comunicação ocorre em um plano musical não verbal (sonoro-musical), essa técnica pode desempenhar a função importante de ser usada como uma forma de diálogo pré-verbal, típica das primeiras interações adulto-criança. Tais interações devem ser feitas diretamente com processos de "sintonização afetiva" descritos por Stern (1985), e de comunicação emocional direta. Os elementos constituintes descritos aqui podem ser colocados numa relação de estreita continuidade, com algumas características já mencionadas na presente contribuição de sistemas de música interativos-reflexivos. Em particular, são sistemas adaptativos e intuitivos e, de forma semelhante à natureza extemporânea da improvisação musicoterapêutica, permitem que se aprendam as modalidades de interação enquanto se interage com o sistema. A este respeito, a construção psicológica da corregulação elaborada no âmbito das análises do processo comunicativo diádico é definida como uma contínua descoberta de ação individual que é suscetível de vir a ser modificada continuamente pelas ações do parceiro (FOGEL, 2000). A corregulação indica, portanto, a existência de uma contínua coordenação compartilhada entre os membros da díade; define-se, assim, a qualidade do processo comunicativo. Assim, como as características de SMIRs de agnosticismo e semelhança ou efeito de espelho (PACHET, 2006) favorecem tais tipos de interações diádicas (ADDESSI, 2014), o musicoterapeuta usa a música improvisada criativamente para promover e manter constantemente o contato com a criança, criar um clima de confiança para apoiar e responder, em tempo real, às necessidades específicas da criança (BUNT, 2003). E é sempre útil lembrar que, na prática de musicoterapia, a presença de referências musicais predeterminadas é mínima, e a improvisação é concebida como uma oportunidade de "encontrar e conhecer o outro através de formas sonoras geradas espontaneamente e em conjunto" (PAVLICEVIC, 2000, p. 272).

\section{Conclusões e etapas futuras}

Recentemente, enfatizou-se que a pesquisa atual sobre os efeitos benéficos da música são de grande importância em todas as áreas de assistência da saúde e social 
(MACDONALD, 2013). Isso dá a oportunidade de adotar uma abordagem multidisciplinar que facilite uma abordagem plural da pesquisa e da prática, incorporando as contribuições teóricas de cada disciplina envolvida, como no caso da escola, para responder às necessidades que sempre mais evidenciam a coexistência de elementos que envolvem os planos emocionais-afetivos-relacionais e sociais. Se, por conseguinte, o design das intervenções adequadas parece basear-se na apreciação das especificidades de cada figura profissional, vale a pena recordar que a especificidade da intervenção de musicoterapia é a centralidade da observação e análise do processo interpessoal que se desenvolve principalmente através de som e da música.

Dentro do projeto MIROR, várias pesquisas experimentais foram realizadas com aplicações da plataforma nos campos da musicoterapia e reabilitação (ANAGNOSTOPOULOU, 2012; LEONI, 2011; BONFIGLIOLI; ADDESSI, no prelo b) que mostraram como a interação reflexiva com as aplicações MIROR pode promover a criatividade musical e a aprendizagem musical em uma área de musicoterapia e no uso dos princípios da musicoterapia em contextos educacionais. A partir desses experimentos e pesquisas com crianças e tecnologias reflexivas surgiram propostas de atividades criativas e expressivas que podem ser realizadas em contextos educacionais, terapêuticos, de inclusão e de atendimento (ver BONFIGLIOLI, 2015; FERRARI; ADDESSI, 2016). Novos aplicativos MIROR estão sendo processados. Em particular, estamos trabalhando com o Royal Institute of Technology e o Royal College of Music, em Estocolmo, para projetar o MIROR-MultiModal, um software que aplica o paradigma da reflexividade à interação musical, motora e visual. Aqueles que usarão aplicativos do MIROR poderão se beneficiar dos resultados da pesquisa até agora e, por outro lado, podem ajudar a enriquecer as experiências com novos problemas, ideias, soluções, pesquisas e experimentação, aos processos de pesquisa já realizados com o Projeto MIROR, sobre o uso de tecnologias reflexivas em contextos educacionais formais e não formais, musicoterapia, inclusão e bem-estar.

Os resultados desses estudos possibilitam a criação de novas aplicações dentro do projeto europeu LINK-Learning In a New Key, cujo objetivo é implementar programas de desenvolvimento profissional para professores dos ensinos fundamental e médio, focados na educação emocional e social de jovens com várias formas de necessidades (ADDESSI; BONFIGLIOLI; CLOUGH, 2016). Esses programas, voltados para professores de educação musical e outras matérias curriculares, preveem a utilização e a integração de práticas musicais, musicoterápicas e artísticas nas atividades curriculares. As ferramentas desenvolvidas no âmbito do projeto MIROR, com base em parâmetros de interação reflexiva para observação do estado do fluxo, serão usadas com técnicas de observação da musicoterapia para estudar estados de bem-estar relacionados a experiências de aprendizagem vivenciadas nas salas de aula por professores junto com crianças e adolescentes durante as atividades musicais que serão realizadas dentro do projeto LINK. 


\section{Referências}

ADDESSI, A. R. The musical dimension of daily routines with under-four children during diaper change, bedtime and free-play. In: ILARI, B.; GLUSCHANKOF, C. (a cura di). Music in the early years: Research, theory and practice. Early Child Development and Care, v. 179, n. 6, p. 747-768, 2009. Special Issue.

Child/machine interaction in reflexive environment. The MIROR platform. In: BRESIN, R. (a cura di). Proceedings of the Sound and Music Computing Conference 2013. Berlin, Verlag, 2013. p. 92-102.

Developing a theoretical foundation for the reflexive interaction paradigm with implications for training music skill and creativity. Psychomusicology: Music, Mind, and Brain, v. 24, n. 3, p. 214-230, 2014.

La creatività musicale e motoria dei bambini in ambienti riflessivi: pratiche didattiche con la piattaforma MIROR. Bologna: Bononia University Press, 2015.

ADDESSI, A. R.; PACHET, F. Experiments with a musical machine. Musical style replication in 3/5 year old children. British Journal of Music Education, v. 22, n. 1, p. 21-46, 2005.

Young children confronting the Continuator, an interactive reflective musical system, Musicae Scientiae, v. 10, n. 1 (Suppl.), p.13-39, 2006.

ADDESSI, A. R.; BONFIGLIOLI, L.; CLOUGH, N. The impact of teachers' music training on flow experience and emotion regulation of students and teachers in the framework of the LINK project. In: NOGUEIRA, M. (Ed.). Anais do XII SIMCAM Simpósio International de Cognição e Arts Musicais. Revista ABEM, p.113-120, 2016.

ADDESSI, A. R.; FERRARI, L.; CARUGATI, F. The Flow Grid: A technique for observing and measuring emotional state in children interacting with a flow machine. Journal of New Music Research, v. 44, n. 2, p. 129-144, 2015.

ADDESSI, A. R.; MAFFIOLI, M.; ANELLI, F. The MIROR platform for young children's music and dance creativity. Reflexive interaction meets body-gesture, embodied cognition, and Laban educational dance. Perspectives. Journal of the Early Childhood Music and Movement Association, v. 10, n. 1, p.09-18, 2015.

ADDESSI, A. R.; ANELLI, F.; BENGHI, D.; FRIBERG, A. Child-computer interaction at the beginner stage of music learning: Effects of reflexive interaction on children's musical improvisation. Frontiers in Psychology, v. 8, n. 650, 2017. DOI: <10.3389/fpsyg.2017.00065>.

ADDESSI, A. R.; ANAGNOSTOPOULOU, C.; NEWMAN, S.; OLSSON, B.; PACHET, F.; VOL- 
PE, G.; YOUNG, S. MIROR Project Final Report, 2013. Disponível em: <http://www.mirorproject.eu/download/Final-Report_3rd-Year.pdf>.

ALEXAKIS, A.; KHATCHATOUROV, A.; TRIANTAFYLLAKI, A.; ANAGNOSTOPOULOU, C. Measuring musical creativity advancement. In: BRESIN, R. (Ed.). Proceedings of the Sound and Music Computing Conference. Berlin: Logos Verlag, 2013.

ANAGNOSTOPOULOU, C.; ALEXAKIS, A.; TRIANTAFYLLAKI, A. A computational method for the analysis of musical improvisations by young children and psychiatric patients with no musical background. In: CAMBOUROPOULOS, E.; TSOUGRAS, C.; MAVROMATIS, P.; PASTIADIS, K. (a cura di). Proceedings of the 12th International Conference on Music Perception and Cognition and the 8th Triennial Conference of the European Society for the Cognitive Sciences of Music. Thessaloniki, Greece, 2012, p. 64-68.

ANZIEU, D. Les enveloppes psychiques. Paris : Dunod, 1996.

BARONI, M. Elogio della musicologia sistematica. Rivista Italiana di Musicologia, v. 2, n. 20, p. 332-352, 1985.

Suoni e significati. Attività espressive nella scuola. Torino: EDT, 1997.

BEEBE, B.; LACHMANN, F. M. Infant Research e trattamento degli adulti. Milano: Raffaello Cortina Editore, 2003.

BONFIGLIOLI, L. La componente MIROR-Impro nel setting musicoterapico: un percorso di musicoterapia di gruppo con i bambini. In: ADDESSI, A. R. (a cura di). La creatività musicale e motoria dei bambini in ambienti riflessivi: pratiche didattiche con la piattaforma MIROR. Bologna: Bononia University Press, 2015. p. 139-152.

L'integrazione sociale attraverso la comunicazione sonoro-musicale: un percorso di musicoterapia con i bambini di tre anni. Infanzia, v. 2, p. 130-132, 2008.

BONFIGLIOLI, L.; ADDESSI, A. R. Improving communication through reflexive musical interaction in therapeutic setting with children. (no prelo a).

New technologies in music therapy with children: A pilot study with the MIROR platform, and adaptive system based on the "reflexive interaction" paradigm (no prelo b).

BRUNER, J. Child's talk: Learning to use language. Trad. it. Armando. New York: W.W. Norton \& Co., 1983.

BRUSCIA, K. Improvisational models of music therapy. Springfield, Illinois: Charles Thomas Publisher, 1987. 
Defining Music Therapy. New York: Barcelona Publishers, 1998.

BUNT, L. Musicoterapia, un'arte oltre le parole. Edizioni Kappa: Roma, 1997.

Music therapy: A resource for creativity, health and well-being across the lifespan. In: ODENA, O. (a cura di). Musical creativity: Insights from music education research. Farnham, UK: Ashgate, 2012. p.165-181.

BUNT, L 'Music Therapy with Children: A Complementary Service to Music Education?' British Journal of Music Education, vol.20, no.2, Cambridge University Press, 2003, p. 179-195.

CANEVARO, A. Mediatori efficaci. Appunti sulle politiche sociali, v. 6 p. 01-05, 2007.

CARDOSO de ARAÚJO, R.; ADDESSI, A. R. Um estudo sobre a improvisação musical de crianças num contexto musical interativo/reflexivo. Música em Contexto, v. 8, n. 1, p. 76-91, 2014.

CATERINA, R.; BUNT, L. Musicoterapia; In: NATTIEZ, J. J.; BENT, M.; DALMONTE, R.; BARONI, M. (a cura di). Enciclopedia della musica, Il sapere musicale. V. 2. Torino: Einaudi, 2002, p. 419-442.

CROSS, I. Musicality and the human capacity for culture. Musicae Scientiae, v. 12, p.147167, 2008.

CSIKZSENTMIHALYI, M. Creativity. Flow and the Psychology of discovery and invention. New York: Harper Collins Edition, 1996.

DELALANDE, F. Le condotte musicali. Bologna: Clueb, 1993.

DELIĖGE, I. Similarity perception _-_C Categorization _-_C Cue abstraction. Music Perception, v. 18, p. 233-243, 2001.

DISSANAYAKE, E. Antecedents of the temporal arts in early motherinfant interaction. In: WALLIN, N. L.; MERKER, B.; BROWN, S. (a cura di). The origins of music. Cambridge, MA: MIT Press, 2000. p. 389-410.

ENGEL, G. The need for a new medical model: A challenge for biomedicine. Science, v. 19, p. 129-136, 1977.

FERRARI, L.; ADDESSI, A. R. Early exploration of digital sound: Two-three year old children interacting with the MIROR Impro. In: PITT, J.; RETRA, J. (a cura di). MERYC2013. Proceedings of the European Network of Music Educators and Researchers of Young Children. The Hague (Finland), 2013. p. 359-368. 
. A new way to play music together: The Continuator in the classroom setting. International Journal of Music Education, v. 32, p. 171-184, 2014.

Suonare con il MIROR-Impro: potenzialità inclusive nei sistemi interattivi riflessivi musicali. In: EMILI, E. ANGELO (a cura di). L'aula possibile. Firenze: Libri Liberi, 2016. p 104-118.

FOGEL, A. Developing through relationships: Origins of communication, self and culture. London: Harvester Wheatsheaf, 1993.

Oltre gli individui: un approccio storico-relazionale alla teoria e alla ricerca sulla comunicazione. In: GENTA, M. L. (a cura di). Il rapporto madre-bambino. Roma: Carocci, 2000. p. 123-161.

FOLKESTAD, G.; HARGREAVES, D. J.; LINDSTRÖM, B. Compositional strategies in computer-based music-making. British Journal of Music Education, v. 15, n. 1, p. 83-97, 1998

GALILEI, V. Dialogo della musica antica et della moderna. Ed. Roma: Reale Accademia d'Italia, 1934. [Firenze, Marescotti, 1531]

GRATIER, M. Grounding in musical interaction: Evidence from jazz performances. Musicae Scientiae, v. 12, n. 1 (Suppl.), p. 71-110, 2008.

GRATIER, M.; APTER-DANON, G. The musicality of belonging: Repetition and variation in mother-infant interaction. In: MALLOCH, S.; TREVARTHEN, C. (Eds.). Communicative musicality. Oxford, New York, NY: Oxford University Press, 2008. p. 301-327.

IMBERTY, M. La musique creuse le temps. De Wagner à Boulez: Musique, psychologie, psychoanalyse. Paris: L'Harmattan, 2005.

LABAN, R. The mastery of movement. 4. ed. London, UK: Macdonalds \& Evens Limited, 1980.

LEMAN, M. Embodied music cognition and mediation technology. Cambridge, MA: MIT Press. 2007.

Systematic musicology at the crossroads of modern music research. In: SCHNEIDER, A. (Ed.). Systematic and comparative musicology: Concepts, methods, findings Hamburger. Jahrbuch fur Musikwissenschaft. V. 24. Frankfurt am Main: Peter Lang, 2008. p. 89-115.

LEONI, M. "No stato io solo!". Un'esperienza con la piattaforma MIROR (Musical Interac- 
tion Relying On Reflexion) in un contesto educativo speciale. Tesi di laurea in Metodologia dell'Educazione Musicale, Corso di Laurea in Scienze della Formazione Primaria, relatore Prof.ssa Anna Rita Addessi, corr. Dott.ssa Luisa Bonfiglioli, Università di Bologna, 2011.

MACDONALD, R. A. R. Music, health, and well-being: A review. International Journal of Qualitative Studies on Health and Well-Being, v. 8, 2013. Disponível em: <http://doi. org/10.3402/qhw.v8i0.20635>.

MALLOCH, S.; TREVARTHEN, C. (Eds.). Communicative musicality. Exploring the basis of human companionship. Oxford: Oxford University Press, 2009a.

MALLOCH, S.; TREVARTHEN, C. Musicality: Communicating the vitality and interests of life; In: MALLOCH, S.; TREVARTHEN, C. (a cura di). Communicative musicality. Exploring the basis of human companionship, Oxford: Oxford University Press, 2009b, p. 01-10.

MARGULIS, E. H. On repeat. How music plays the mind. New York: Oxford University Press, 2014.

MCPHERSON, G. From child to musician: Skill development during the beginning stage of learning an instrument. Psychology of Music, v. 33, p. 05-35, 2005.

MITHEN, S. The singing Neanderthals: The origin of music, language, mind and body. London: Weidenfeld \& Nicolson, 2005.

NADEL, J.; BUTTERWORTH, G. (Eds.). Imitation in infancy. Cambridge: Cambridge University Press, 1999.

NADEL, J. Imitation and imitation recognition: Functional use in preverbal infants and nonverbal children with autism. In: MELTZOFF, A. N.; PRINZ, W.(Eds.) The imitative mind: Development, evolution and brain bases. Cambridge, UK: Cambridge University Press, 2002, p. 42-62.

PACHET, F. Musical interaction with styl. Journal of New Music Research, v. 32, n. 3, p. 333-341, 2003.

On the design of Flow Machine. In: TOKORO, M. (a cura di). A learning zone of one's own: Sharing representations and flow in collaborative learning environments. The Netherlands: IOS Press, 2004. p. 111-134.

Creativity studies and musical interaction. In: DELIĖGE, I.; WIGGINS, G. A. (a cura di), Musical creativity. Hove: Psychology Press, 2006. p. 347-358. 
PACHET, F.; ROY, P.; BARBIERI, G. Finite-Length Markov processes with constraints. Proceedings of IJCAI 2011, Barcelona, July 2011.

PAPOUŠEK, M. Communication in early infancy: An arena of intersubjective learning. Infant Behavior and Development, v. 30, p.258-266, 2007.

PAVLICEVIC, M. Improvisation in Music Therapy: Human Communication in Sound. Journal of Music Therapy, Volume 37, Issue 4, 1 December 2000, p. 269-285

POSTACCHINI, P. L.; RICCIOTTI, A.; BORGHESI, M. Lineamenti di musicoterapia. Roma: Carrocci, 1998.

POSTACCHINI, P. L.; BORGHESI, M.; RICCHIOTTI, A. Musicoterapia. Roma: Carocci, 2004.

RICCI BITTI, P. E. (Ed.). Regolazione delle emozioni e artiterapie. Roma: Carocci, 1998.

RIZZOLATTI, G.; FADIGA, L.; FOGASSI, L.; GALLESE, V. From mirror neurons to imitation: Facts and speculations. In: MELTZOFF, A. N.; PRINZ, W. (a cura di). The imitative mind. Development, evolution, and brain bases. New York, NY: Cambridge University Press, 2002. p. 247-266.

ROWE, V.; TRIANTAFYLLAKI, A.; ANAGNOSTOPOULOU, C. Young pianists exploring improvisation using interactive music technology. International Journal of Music Education, v. 33, p. 113-130, 2014.

RUWET, N. Méthodes d'analyse en musicologie. Revue belge de Musicologie, v. 20, p. 65-90, 1966.

SAWYER, K. R. Improvised conversation: Music, collaboration and development. Psychology of Music, v. 27, p. 192-216, 1999.

STERN, D. N. Il mondo interpersonale del bambino. Torino: Boringhieri, 1985.

The present moment in psychotherapy and in everyday life. New York, NY: Norton, 2004.

TAFURI, J. L'educazione musicale. Teorie, metodi, pratiche. Torino: EDT, 1995.

Processes and teaching strategies in musical improvisation with children. In: DELIĖGE, I.; WIGGINS, G. A. (a cura di). Musical creativity. Hove: Psychology Press, 2006. p. 134-158. 
TOIVIAINEN, P. (Ed.) Similarity perception in listening to music. Musicae Scientiae, v. 11, Special Issue, 2007.

TRIANTAFYLLAKI, C.; ANAGNOSTOPOULOU, A.; ALEXAKIS, A. An exploratory study of young children's technology-enabling improvisation. In: CAMBOUROPOULOS, E.; TSOUGRAS, C.; MAVROMATIS, P.; PASTIADIS, K. (a cura di). Proceedings of the 12th International Conference on Music Perception and Cognition and the 8th Triennial Conference of the European Society for the Cognitive Sciences of Music. Thessaloniki, Greece, 2012. p.1009-1015.

VOLPE, G.; VARNI, G.; ADDESSI, A. R.; MAZZARINO, B. BeSound: Embodied reflexion in childhood. In: Proceedings of the 11th International Conference on Interaction Design and Children (IDC2012). New York, NY, USA, ACM, 2012. p. 172-175.

VYGOTSKY, L. S. Thought and language. Cambridge, MA: MIT Press, 1962.

WALLERSTEDT, C; LAGERLOF, P. Exploring turn-taking in children's interaction with a new music technology. HeKupu, v. 2, n. 5, p. 20-31, 2011.

WIGRAM, T. Improvvisazione. Metodi e tecniche per clinici, educatori e studenti di musicoterapia. Roma: Ismez, 2004.

WIGRAM, T.; PEDERSEN, I. N.; BONDE, L. O. Guida generale alla musicoterapia. Teoria, pratica clinica, ricerca e formazione. Roma: Ismez, 2003.

WHEELER, B. L. (a cura di). Music therapy handbook. New York, NY: Guilford Press, 2015.

ZAGATTI, F. La danza educativa. Principi metodologici e itinerari operativi. Bologna: Mousikè Progetti Educativi, 2004.

ZATORRE, R. J. Beyond auditory cortex: Working with musical thoughts. In: OVERY, K.; PEREZ, I.; ZATORRE, R. J.; LOPEZ, L.; MAJNO, M. (Eds.). The Neurosciences and Music-IV. Learning and Memory. Annals of the New York Academy of Sciences, 2012, p. 222-228. 